\title{
To the problem of the secondary CMB anisotropy separation
}

\author{
Oleg Verkhodanov ${ }^{1, \star}$, Dmitrij Solovyov ${ }^{2, \star \star}$, Elena Majorova ${ }^{1, \star \star \star}$, and Margarita \\ Khabibullina ${ }^{1, \star \star \star \star}$ \\ ${ }^{1}$ Special Astrophysical Observatory, Russian Academy of Sciences, Nizhnii Arkhyz, 369167 Russia \\ ${ }^{2}$ St. Petersburg Branch of the Special Astrophysical Observatory of the Russian Academy of Sciences, \\ St.Petersburg, Russia 196140
}

\begin{abstract}
We study contribution to the secondary anisotropy maps of cosmic microwave background $(\mathrm{CMB})$ radiation which difficult to account for faint sources. Two effects are investigated. They are the Sunyaev-Zeldovich effect connected with the inverse Compton scattering of $\mathrm{CMB}$ photons on hot electrons of cluster of galaxies, and contamination of the background by weak extragalctic sources. First, we study fields of the Planck CMB maps around radio sources of the RATAN-600 catalog. We see weak microwave sources which make an additional contribution to the secondary anisotropy on angular small scales $\left(<7^{\prime}\right)$. An algorithm for selecting candidate objects with the SunyaevZeldovich effect was proposed, based on the use of data on the radio spectral indices and the signal in cosmic-microwave background maps. Second, applying the stacking method, we examine the areas of the CMB maps, constructed according to the Planck Space Observatory data in the neighborhood of different populations of radio sources and giant elliptical galaxies. The samples of objects include giant radio galaxies (GRG), radio sources, selected by the radio spectral index and redshift, as well as the gammaray bursts, used as a secondary comparative sample. The signal from this objects exists on CMB maps and its difference in the neighborhood of GRGs from the other types of objects was discovered.
\end{abstract}

\section{Introduction}

Publishing of the microwave maps restored from the millimeter and submillimeter observations of the NASA WMAP ${ }^{1}$ (Wilkinson Microwave Anisotropy Probe) [1] and ESA Planck ${ }^{2}$ [2] opened a new era in the research of the extended foreground emission and the extragalactic sources. The key role in last five years was played by the Planck Space Observatory and the full sky maps which were constructed based on the results of its work at nine frequencies of the microwave range: 30, 44, 70, 100, 143, 217, 353, 545, and $847 \mathrm{GHz}$. The maps of the Planck satellite of the European Space Agency were opened to the scientific community in 2013 (and the second release in 2015) and at once became an

\footnotetext{
${ }^{\star}$ e-mail: vo@sao.ru

$\star \star$ e-mail: drukhi@ya.ru

$\star \star \star$ e-mail: len@sao.ru

$\star \star \star \star$ e-mail: rita@sao.ru

${ }^{1}$ http://lambda.gsfc.nasa.gov

${ }^{2}$ http://www. cosmos.esa.int/web/planck
} 
effective means for solving many problems of galactic and extragalactic astronomy. Moreover, the catalogs of the discovered galactic and extragalactic sources of the millimeter and submillimeter radiation were presented in the first data release. The measurements of radiation fluxes were conducted using the High Frequency Instrument (HFI) with frequency bands of $100 \mathrm{GHz}$ and higher, and the Low Frequency Instrument (LFI) with frequency bands lower than $100 \mathrm{GHz}$. The resolution of the maps at frequencies higher than $100 \mathrm{GHz}$ amounted to about $5^{\prime}$. Although the Planck mission is secondary (by the launch date) relative to the other space mission, WMAP, it has better observational characteristics, which allow scientists to use it for search and investigation of weak radio sources in themicrowave range. Among the most important astrophysical products of the Planck mission, we can mention (1) table of cosmological parameters; (2) cosmic microwave background (CMB) radiation maps; (3) channel data maps (temperature anisotropy and polarization); (4) foregrounds maps; (5) secondary anisotropy maps; and (6) catalogs of millimeter and submillimeter sources. Among the advantageous features of Planck in comparison with the WMAP, let us note the higher resolution (three times higher), higher response (ten times higher), and nine frequency bands, which allowed the radiation components to be separated. The Planck Legacy Archive ${ }^{3}$ (PLA) contains both the maps of radiation components and the lists of the detected objects. PLA contains the data base of sets of maps and we mention some of them below. First, four CMB maps of cosmic microwave background (CMB) radiation restored by 4 component separation methods, respectively. They are (a) internal linear combination in spherical wavelets domain - needlets (NILC), (b) template fitting using data at different frequencies to estimate by the expectation maximazation algorithm (Spectral Estimation Via Expectation Maximazation - SEVEM), (c) the method realized in the sperical harmonic domain of amplitude matching of the CMB spectral parameters and background components (Spectral Matching Independent Component Analysis - SMICA), and (d) the Bayesian separation of components in the pixel space by matching a parametric model (Commander).

The second group of PLA contains sky maps at observational bands at 30, 44, 70, 100, 143, $217,353,545,857 \mathrm{GHz}$, where channels from 30 to $353 \mathrm{GHz}$ include the Q,U-polarization data too. The $3 \mathrm{~d}$ group of maps are sky maps of diffent foregrounds, e.g. CO emission maps, dust, galactic low frequency emission (synchrotron plus free-free), zodiac light maps, etc. Also, there are maps of gravitational lensing, $\mathrm{y}$-comptonization maps, and separately, masks used for restored different CMB temperature and polarization maps.

\section{Secondary anisotropy maps}

There exist several basic effects which acted on the CMB fluctuations and can be discussed in terms of the secondary anistropy. Among them are the intergated Sachs-Wolfe effect [3], the SunyaevZeldovich effect [4], reionization, gravitational lensing of CMB, contamination of microwave background with extragalactic radio sources. The Planck observatory produced some secondary anisotropy maps for the astronomical community use. In Figures 1 and 2 are shown the Wiener-filtered lensing potential distribution on the sky [5] and the reconstructed the Planck all-sky y-map in Compton parameter units [6], respectively.

In Fig. 3, we demonstate positions on the sky of microwave sources from Planck calalogs at different frequencies. The used Second Planck Catalogue of Compact Sources [7] is the catalogue of sources detected from the full duration of Planck operations. It consists of compact Galactic and extragalactic objects detected over the entire sky. The total number of sources in the catalogue ranges from 1560 at $30 \mathrm{GHz}$ up to 48181 sources at $857 \mathrm{GHz}$.

\footnotetext{
${ }^{3}$ http://pla.esac.esa.int/pla/
} 


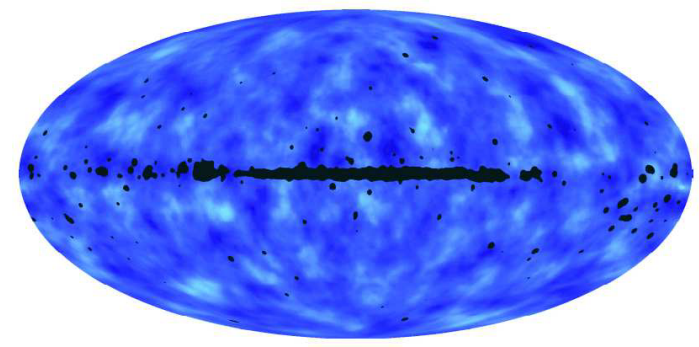

Figure 1. The Wiener-filtered lensing potential estimate with minimal masking (using the NILC component separated map). From [5].

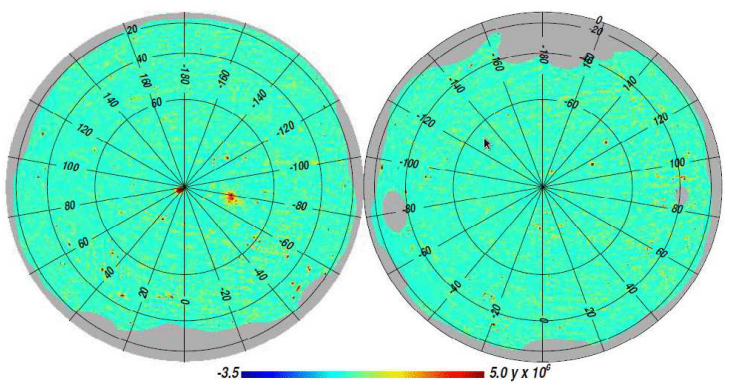

Figure 2. The orthographic projection of the reconstructed Planck all-sky y-map in Compton parameter units. Positive sources in the map correspond to clusters and super-clusters of galaxies with strong tSZ emission. The Coma and Virgo clusters are clearly visible near the north Galactic pole. The region of strongest contamination from Galactic foreground emission in the Galactic plane has been partially masked. From [6].

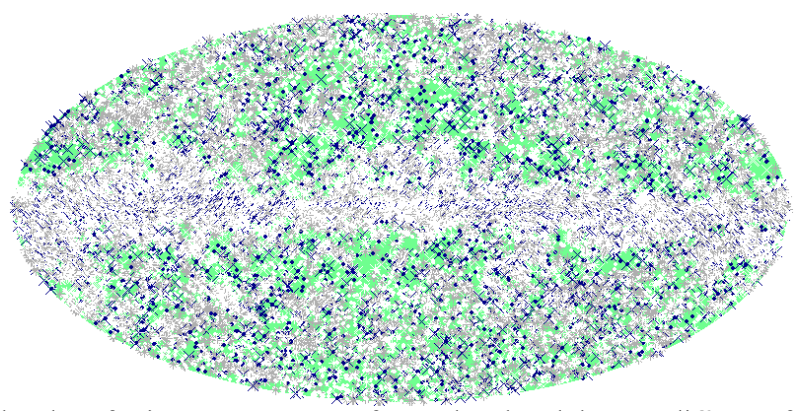

Figure 3. Positions on the sky of microwave sources from Planck calalogs at different frequencies. 


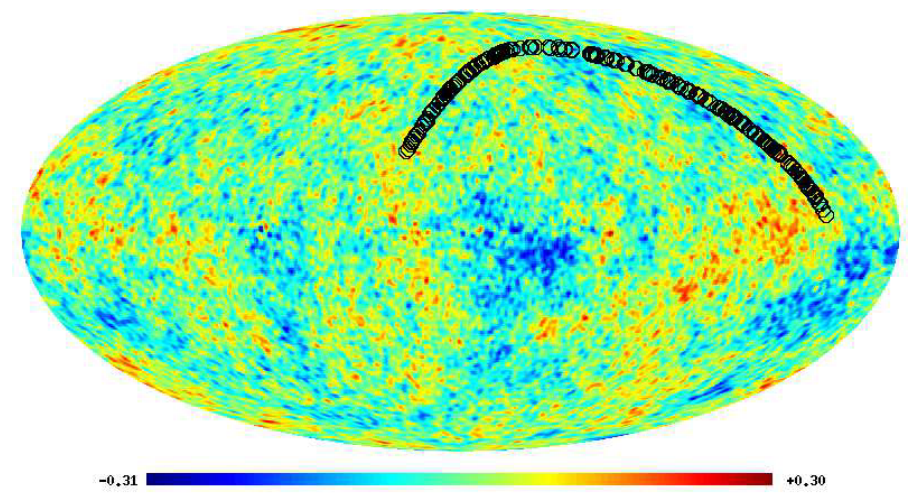

Figure 4. Positions of RCR radio sources on a CMB map (the range of coordinates is $7^{h}<\mathrm{RA}<17^{h}$, Dec $\sim 5^{\circ}$ ).

\section{RCR-sources on the Planck maps}

One of the main problems of the sensitive radio surveys data analysis is the presence of faint sources at the noise level $(\mathrm{S} / \mathrm{N} \lesssim 3)$ which make additional contribution to the angular power spectrum at small angular scales (high multipoles $\ell$ ). This situation is also observed in Planck CMB maps when we consider a signal at $\ell>2000$. Another problem arose for the Planck data is the missed clusters of galaxies which are observed with the Sunayev-Zeldovich effect at multifrequency maps. Number of expected SZ-sources contributed to the secondary anistropy was larger than 20000 (expected number of clusters of galaxies), but the presented catalog contains 1653 objects [8].

We use the RCR (RATAN Cold Refined) catalog $[9,10]$ to look for any response from RC-source direction in millimeter and submillimeter wave length ranges. The RC catalog was obtained during the Cold experiment [9] conducted on the RATAN-600 radio telescope in the 1980s at $3.94 \mathrm{GHz}$. The horizontal and vertical resolutions $60^{\prime \prime}$ and, $15^{\prime}$, respectively. To improve the coordinates and flux densities of the RC catalog sources, seven additional cycles of observations were carried out in the strip of sky corresponding to the Cold survey at several frequencies in 1987-1999, and the data obtained in 1980-1981 were reprocessed for right ascensions $7^{h}<\mathrm{RA}<17^{h}$ and declination Dec $\sim 5^{\circ}$ (Fig. 4). This yielded a list of 551 objects identified with objects in the NVSS catalog with a positional accuracy of $\sim 0.1^{\prime \prime}$. The limiting sensitivity in the RCR catalog was $\sim 10 \mathrm{mJy}$.

Using properties of the CMB signal in the fields of RCR-sources, we prepared some diagrams to explore contamination of the background [11]. Figure 5 shows a plot of radio spectral index $\alpha$ versus CMB response $T_{p}$ corresponding to the thermodynamical temperature on the Planck CMB SMICA map. Note that the $\alpha-T_{p}$ diagram is interesting in that we can distinguish groups of radio sources with correlated parameters that are basically determined by the 'spectral index — redshift' and 'flux density (here temperature) - redshift' relations. Before constructing the diagram, we removed the low-order harmonics $(\ell<20)$, which mainly include the statistically anisotropic signal from the CMB map (Fig. 6). In principle, it is possible to eliminate harmonics with $\ell \leq 220$, before the first CMB peak, but it is important to remove precisely data with $\ell \leq 20$ after which the map becomes Gaussian; for example, features such as the Cold Spot [12] disappear. We can distinguish four regions in the diagram on Fig. 5 (A, B, C, D), which correspond to the positions of peaks on histograms of the parameters of the objects: regions $\mathrm{A}, \mathrm{B}$, and $\mathrm{C}$ correspond to the position of the main peak in the histogram of spectral indices (Fig. 7), and region D corresponds to the second peak in the distribution of the spectral indices for the flat-spectrum sources. The histogram of the radio spectral indices has a standard two-peaked distribution determined by the number of objects with steep and flat spectra, and 


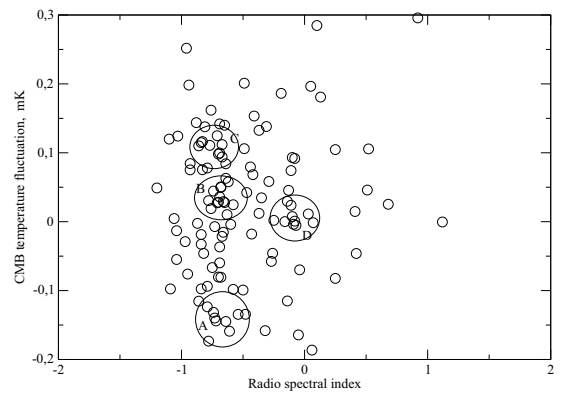

Figure 5. Plot of radio spectral index vs. CMB signal for all the RCR catalog sources. The CMB signals are given in thermodynamical units. The circles mark four groups of objects (A, B, C, D) corresponding to peaks in the histograms and discussed in the text.

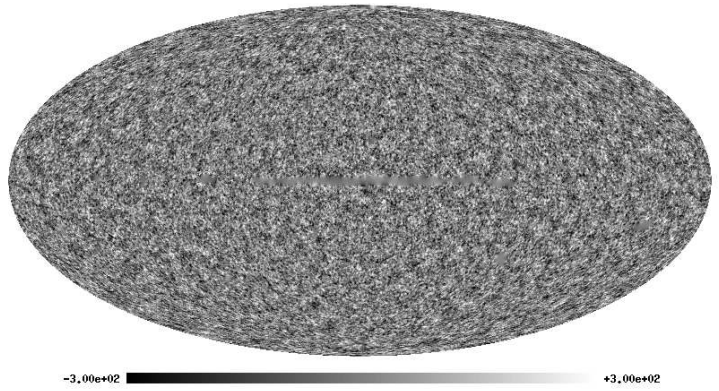

Figure 6. CMB SMICA map for the 2015 Planck data release used in this study for our analysis of the distribution of the CMB fluctuation signal. The low-order multipoles $\left(\ell \leq 20\right.$, i.e., the signal on angular scales larger than $8^{\circ}$, which mainly includes the statistically anisotropic background) were first removed

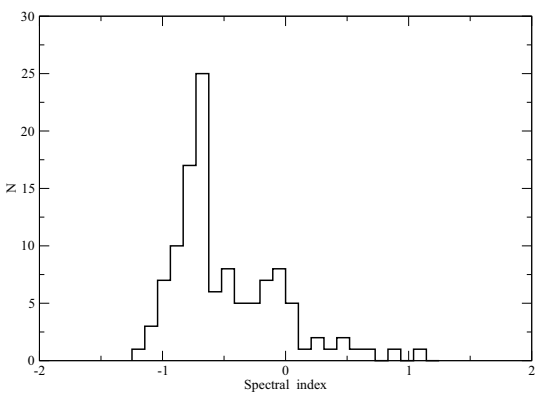

Figure 7. Distribution of spectral indices of all RCR catalog sources at $1.4 \mathrm{GHz}$. 

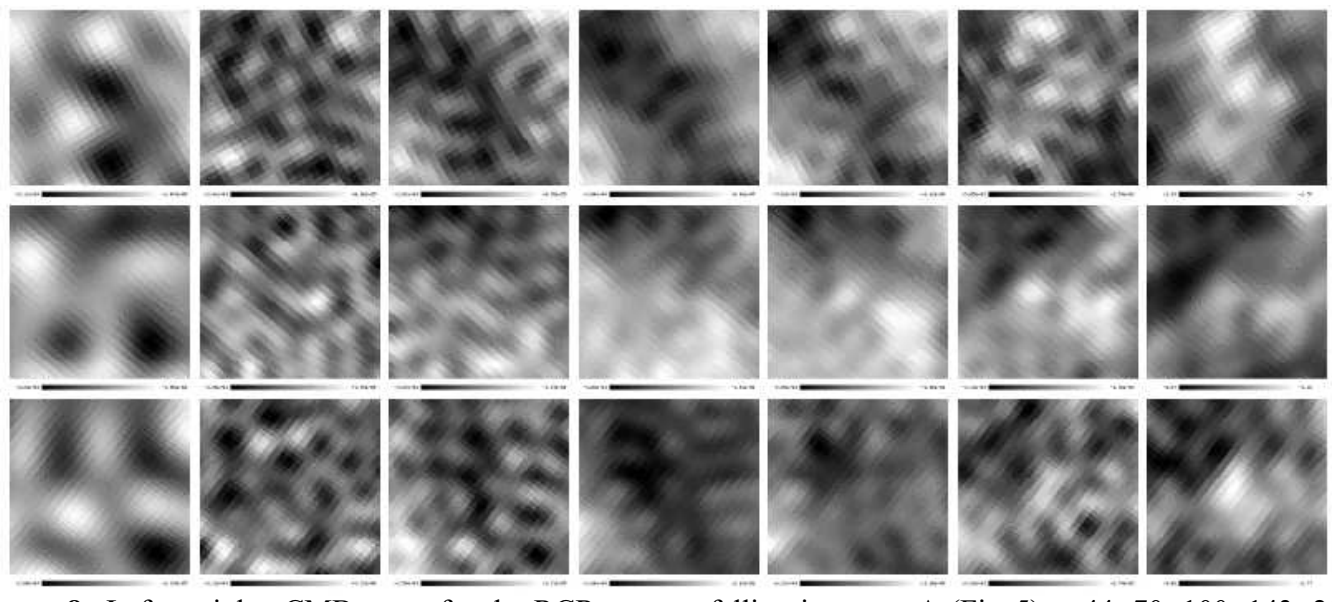

Figure 8. Left to right: CMB maps for the RCR sources falling in group A (Fig. 5) at 44, 70, 100, 143, 217 , 353 , and $545 \mathrm{GHz}$, respectively, according to the 2015 Planck data. For the $\mathrm{S}-\mathrm{Z}$ effect at the Planck frequencies, a dip at $v<217 \mathrm{GHz}$ and a positive peak at $v>217 \mathrm{GHz}$ are expected. From top to bottom: the radio sources $092445+050344,112437+045618$, and $151054+045410$. The size of the images is $0.5^{\circ} \times 0.5^{\circ}$.

described. The main values of the signal in the CMB map are concentrated near zero, but from the positive side: the median CMB signal calculated using the data for all the studied sample sources is $+0.02527 \mathrm{mK}$.

Field A in Fig. 5, which corresponds to radio sources with negative responses exceeding the expected $1 \sigma$ level and having extreme slopes in their radio spectra, $\alpha=0.6-0.8$. The relatively low negative level of the background on the CMB map could be due to an additional contribution to the inhomogeneity of the Sunyaev-Zeldovich effect, enhanced during the separation of the components and reconstruction of the CMB signal. This effect is also suggested by the existence of a radio source in this region, which is a sign of an accumulation of material (ultimately a cluster of galaxies). Figure 8 presents the maps of three RCR sources from field A at various frequencies as example candidates for objects displaying the Sunyaev-Zeldovich effect.

\section{Response from radio galaxies on the Planck microwave maps}

We examined the response of an average object, obtained by stacking the areas sized $1^{\circ} \times 1^{\circ}$ around the sources from different populations according to the microwave maps of the Planck mission [13]. The main population of objects studied comprises 89 giant radio galaxies with the angular sizes of more than $4^{\prime}$, the linear sizes of which (larger than $1 \mathrm{Mpc}$ ) are confirmed in the literature. For a comparison of GRG properties with the properties of other populations we have studied the subsamples of different classes of objects with the selection by the spectral indices, redshifts, optical morphology (the gE and cD-type galaxies), as well as the gamma-ray bursts. Radio galaxies and gian elliptical galaxies (gE) include following sub-samples: 1) giant radio galaxies ( 89 objects, $>1 \mathrm{Mpc}, \theta>4^{\prime}$ ); 2) distant radio galaxies (2400) [14]; 3) giant elliplical galaxies (gE and cD) from clusters of galaxies (25) [15]; 4) WENSS radio sources in the range of right ascensions: $\left.0^{h} \leq \mathrm{RA}<2^{h} ; 5\right)$ objects from BATSE [16] and BeppoSAX [17] gamma-ray burst catalogs. Applying the stacking method, we examine the areas of the CMB maps, constructed according to the Planck data in the neighborhood of this populations of galaxies. The samples of objects include giant We have studied the topological properties of the 

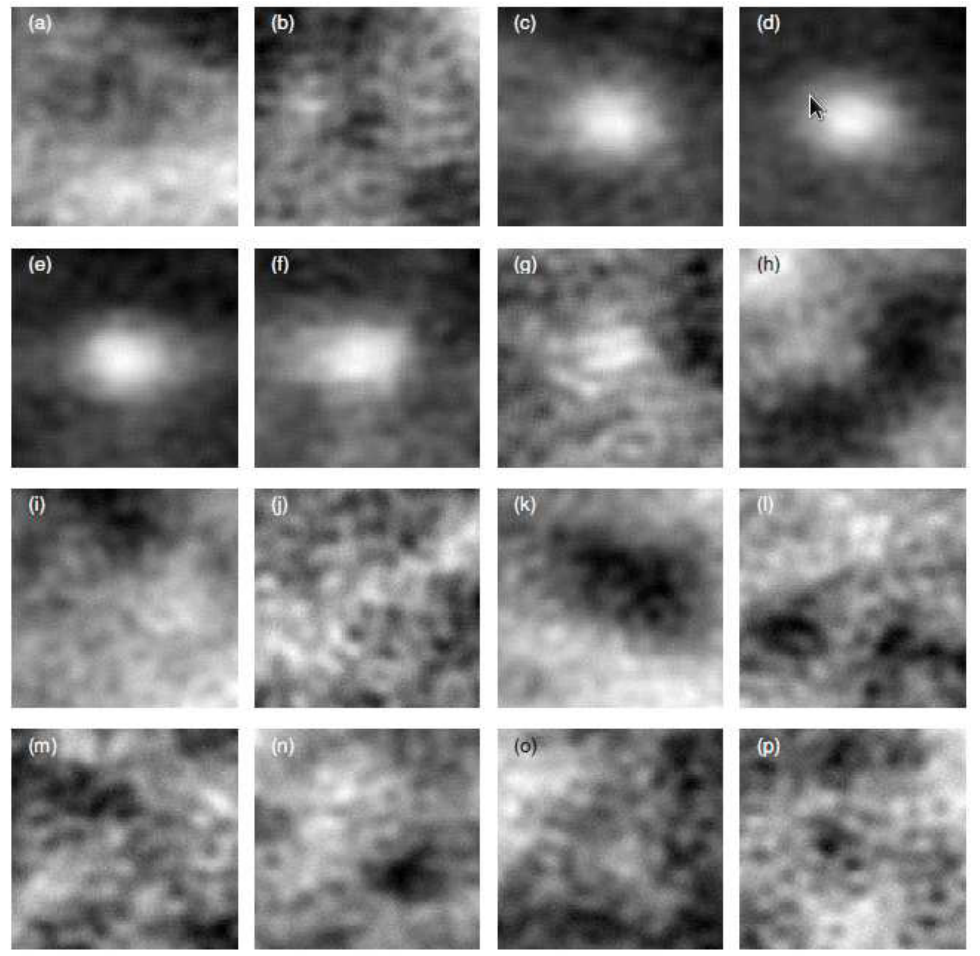

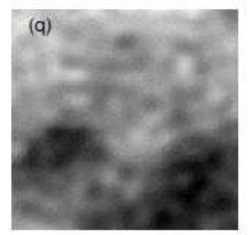

Figure 9. The stacking zones sized $1^{\circ} \times 1^{\circ}$ built from the CMB SMICA Planck 2.02 map data. From left to right and from top to bottom are presented the images of the average maps for the object populations (the number of sample objects is marked in the brackets): (a) GRGs with the angular sizes of $\theta>4^{\prime}$ and linear sizes of $>1 \mathrm{Mpc}$ (89), (b) the gE and CD galaxy types (25), (c) the radio sources (RS) from the WENSS survey with the radio spectral indices $\alpha<-1.1$ (224), (d) WENSS RS with $-1.1 \leq \alpha<-0.75$ (661), (e) WENSS RS with $-0.75 \leq \alpha<-0.5$ (497), (f) WENSS RS with $-0.5 \leq \alpha<0$ (238), (g) WENSS RS with $\alpha \geq 0$ (19), (h) distant RG with the redshifts $0.3 \leq z<0.7$ (1797), (i) HZRG with $0.7 \leq z<1.0$ (205), (j) HZRG with $1.0 \leq z<1.5$ (149), (k) HZRG with $1.5 \leq z<2.0$ (103), (k) HZRG with $2.0 \leq z<2.5$ (77), (1) HZRG with $z \geq 2.5$ (81), (n) the BATSE events with the duration of $t<2 \mathrm{~s}$ (495), (o) BATSE, $t>2 \mathrm{~s}$ (1540), (p) BeppoSAX, $t<2 \mathrm{~s}$ (87), (q) BeppoSAX, $t>2 \mathrm{~s}$ (694). The original CMB data are given in the scale of thermodynamic temperatures.

CMB signal in the neighborhood of the average object of the population, namely, we searched for the presence of the maxima and minima in the average area (Fig. 9). The difference of the signal in the neighborhood of GRGs from the other types of objects was discovered.

\section{Summary}

It is possible to note a few points: 
- There is a positive signal for objects of different radio galaxy popultaion;

- It is possible to increase the number of SZ-objects for several times using searching fields around radio sources;

- There is a positive response in the zone of radio sources which number should be tens time larger than Planck source list;

- All objects are detected within $7^{\prime}$ zones, thus they do not affect the $C(\ell)$ power spectrum at low and intermediate $(\ell<2000)$ multipoles.

For an average GRG in the microwave range, the area around which is built by stacking, it was found that 1) there is a positive signal in the millimeter range; 2) the spectrum in the millimeter range is flat; 3) the signal in the submillimeter range is absent; 4) the topological properties in the average CMB fluctuations map possess special features: a weak insignificant maximum $(<1 \sigma)$ is located in the minimum zone; 5) a significant SZ-effect, related with the scattering of CMB photons by the electrons of the GRG jet plasma [18] is not observed. A comparison with other populations of objects has revealed that given the average microwave properties, the distant radio galaxies with redshift ranges $0.3 \leq z<0.7$.

Acknowledgments. We thank the European Space Agency for their open access to the observations and data in the Planck Legacy Archive. We have used the GLESP package [19] for the analysis of extended emission on a sphere.

\section{References}

[1] C.L. Bennett, D. Larson, J.L. Weiland, et al., Astrophys. J. Suppl. 208, 20 (2013).

[2] Planck Collaboration, Astrophys. Astrophys. accepted; arXiv:1502.01582.

[3] R. K. Sachs and A. M. Wolfe, ApJ 147, 73 (1967).

[4] Ya. B. Zeldovich and R. A. Sunyaev, Astrophys. Sp. Sci. 4, 301 (1969).

[5] Planck Collaboration, Astron. Astrophys. accepted; arXiv:1502.01591.

[6] Planck Collaboration, Astrophys. Astrophys. accepted; arXiv:1502.01596.

[7] Planck Collaboration Astron. Astrophys. accepted; arXiv:1507.02058.

[8] Planck Collaboration Astron. Astrophys. accepted; arXiv:1502.01598

[9] Yu. N. Parijskij, N. N. Bursov, N. M. Lipovka, et al., Astron. Astrophys. Supp. 87, 1 (1991).

[10] O. P. Zhelenkova, N. S. Soboleva, E. K. Majorova, and A. V. Temirova, Astrophys. Bull. 68, 26 (2013).

[11] O.V.Verkhodanov, E.K.Maiorova, O.P.Zhelenkova, et al., Astronomy Reports 60, 630 (2016).

[12] P. D. Naselsky, P. R. Christensen, P. Coles, et al., Astrophys. Bull. 65, 101 (2010).

[13] O.V. Verkhodanov, D.I. Solovyov, O.S. Ulakhovich, and M.L. Khabibullina. Astrophys. Bull. 71, 139 (2016).

[14] M. L. Khabibullina and O. V.Verkhodanov, Astrophys. Bull. 64, 123 (2009).

[15] S. A. Stanford, P. R. Eisenhardt, M. Dickinson, et al., Astrophys. J. Suppl. 142, 153 (2002).

[16] W. S. Paciesas, C. A. Meegan, G. N. Pendleton, et al. Astrophys. J. Suppl. 122, 465 (1999), astro-ph/9903205.

[17] D. Riccia, F. Fioreb, and P. Giommia, Nuclear Physics B - Proc. Suppl. 69, 618 (1999).

[18] S. Colafrancesco, P. Marchegiani, Astron. Astrophys. 535, A108 (2011).

[19] A. G. Doroshkevich, P. D. Naselsky, O. V. Verkhodanov, et al., Int. J. Mod. Phys. 14, 275 (2003). 